\title{
Usability Planner: A Tool to Support the Process of Selecting Usability Methods
}

\author{
Xavier Ferre ${ }^{1}$ and Nigel Bevan ${ }^{2}$ \\ ${ }^{1}$ Universidad Politecnica de Madrid, Campus de Montegancedo, \\ 28660 - Boadilla del Monte (Madrid), Spain \\ xavier. ferre@upm.es \\ ${ }^{2}$ Professional Usability Services, 12 King Edwards Gardens, \\ London W3 9RG, UK \\ mail@nigelbevan.com
}

\begin{abstract}
There is increasing pressure on developers to produce usable systems, which requires the use of appropriate methods to support user centred design during development. There is currently no consistent advice on which methods are appropriate in which circumstances, so the selection of methods relies on individual experience and expertise. Considerable effort is required to collate information from various sources and to understand the applicability of each method in a particular situation. Usability Planner is a tool aimed to support the selection of the most appropriate methods depending on project and organizational constraints. Many of the rules employed are derived from ISO standards, complemented with rules from the authors' experience.
\end{abstract}

Keywords: UCD method selection, development process, usability integration in systems development, standards.

\section{Introduction}

UCD (User Centred Design) approaches and methods for systems development are used to help build systems that are easy to use. While there is general consensus on the need for a user-centred approach to design, there is a great diversity of potential methods. Existing literature on usability methods offers little guidance on which specific methods should be used in a given situation.

In order to adopt a UCD focus in development, usability has to be considered throughout the different development stages. The specific UCD methods to apply at each stage must be selected, depending on the relevant project and organizational constraints. This is a challenge because systems development teams and organizations can experience difficulty when trying to understand UCD methods and their role in the overall development process [6].

Existing advice on UCD method selection starts with the method, rather than the purpose for which the method is used. Senior UCD professionals get acquainted during the development of their career with the different subtleties of each method that make it more advisable in certain situations, but other groups (junior UCD consultants, HCI students or even software developers with some HCI training) face 
important difficulties for choosing the most appropriate methods for a particular project. They need to gather information present in different sources, and they have to deal with different terminology across authors in terms of process descriptions, and in terms of criteria for considering the suitability of each method.

The ISO PAS (Publicly Available Specification) 18152:2003 [4] provides an overall view of the life cycle for developing systems for human use, highlighting the base practices associated with the different processes involved. It does not provide specific guidance on method selection but it serves as an overall process framework where to fit advice on UCD methods integration in the systems development process.

The ISO TR (Technical Report) 16982 [5], on the contrary, specifically addresses the issue of UCD method selection. It does not work with individual methods to be applied in a specific situation, but with categories of them. It has an important shortcoming from a professional perspective, in being "somewhat academic" (according to the Special Interest Group in Software Testing from the British Computer Society [1]). Furthermore, the usage of this technical report is not straightforward, since it requires moving between different tables and gathering all the relevant information that is scattered in the document.

Usability Planner [7] is an online tool that supports the process of UCD method selection. It aims to ease the present difficulties found in considering the different knowledge sources, integrating the overall view in ISO PAS 18152:2003 with the recommendations present in ISO TR 16982, and supplementing them with the authors' own experience.

\section{Method Selection with Usability Planner}

The difficulties traditionally found in UCD method selection arise from the difficulty of dealing with a diversity of issues that may influence the choice of methods. In a common scenario, UCD has already been identified in the organization as necessary for improving the quality of the system to produce, and there is a decision on the typically limited - resources to dedicate to UCD in the project.

In these circumstances only a few UCD methods may be chosen, with the aim of maximizing their impact on the final usability level of the system produced. The sequence for making this choice using Usability Planner is:

1. Establish the overall background to consider: Either a UX (User eXperience) UCD background, or a software engineering (developer) one. Depending on the choice a different set of stages into which to allocate the methods is offered.

2. Identify the specific project stages for which methods are to be selected. At this point the user may optionally prioritize the stages according to business benefits or risk avoidance considerations.

3. Specify the constraints related to the project as a whole, or individual stages of the project, and see the recommended methods.

4. View a summary of the recommendations for the project in the form of a usability plan including the selection of methods for each stage. 
Plan which methods to use to support User Centred Design. Optionally prioritize the project stages where usability will provide most benefit

The steps in selecting methods at each stage of design and development supported by the Usability Planner are:

Which best describes you

C Im a UX professional, researcher or student

- Im a developer

- Which UCD activities would provide the greatest cost-benefits or risk mitigation?

- Which of the potential methods that could be used to achieve each activity would be most appropriate?

The tool has a comprehensive list of all the potential purposes for using UCD methods during systems development, based on ISO PAS 18152 I

For which project stage(s) do you want to plan methods to introduce usability improvements into your project? Select project stage(s) to plan the most appropriate methods to achieve the best practices. Optionally prioritize the stages where usability will provide most benefit

Expand all Collapse all

曰 $\nabla$ 1. Concept

F 1.1 Envisioning opportunities

1. Concept

Identify initial system concept

V 1.2 System scoping

more intormation

Substages:

1.1 Envisioning opportunities

Г 2. Planning

more information

- 1.2 System scoping

ᄃ 3. Understanding needs more information

$\Gamma$ 4. Requirements more information

5. Analyse requirements more intormation

(4) Design/development more information

Best practices

- 1.1 Envisioning opportunities substage best practices

- Identify expected context of use of systems [forthcoming needs, trends and expectations]

- Analyze the system concept [to clarify objectives, their viability and risks]

- 1.2 System scoping substage best practices

- Describe the objectives which the user or user organization wants to achieve

through use of the system.

- Define the scope of the context of use for the system.

Back Next 9

How cost effective is each possible method likely to be?

Specify the constraints that will influence which UCD methods are appropriate in your situation

\section{Project constraints}

$\ulcorner$ Need quick results

- Very restricted budget

V Usability important

$\ulcorner$ Uncertain specification

User constraints

$\sqrt{ }$ Difficult to involve user

№ access to users

ᄃ Some users have disabilities

ᄃ Mostly first time users

Tasks constraints

$\Gamma$ Complex task

ᄃ Many tasks

$\Gamma$ Safety or business critical system

$\Gamma$ Organisational changes needed

Product constraints

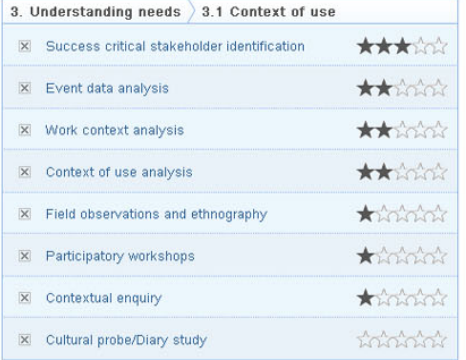

6. Design/development 6.1 High level design

Fig. 1. Three first steps in the usage of the tool for method selection 


\section{Preliminary Evaluation and Conclusions}

When a preliminary prototype [2] was demonstrated to participants at the NordiCHI 2010 conference, all 11 people who tried using the tool were positive about its potential value. One continuing challenge has been to find a sequence that the different types of users find logical for the inter-related decisions that have to be made. Decisions on inter-stage prioritization have been made optional, since some users will not need to address such an issue, and the support that may provided for such prioritization is weaker than the support provided for method selection.

While the tool initially embodies the knowledge of the authors, this will be refined by iterative evaluation. Additionally, the content is customizable, so that any organization with UCD expertise may modify the criteria for method selection in the tool to match their own criteria.

The tool will be offered under an open source license (GNU General Public License, version 3 [3]), so that the community can modify it or further extend it.

Initial responses to the value of the first complete prototype of the tool have been very positive. The next steps include:

1. Refining the set of methods and criteria, and the internal weighting, to find out whether the tool can consistently make similar recommendations to experts.

2. More formal evaluation of the usefulness and usability of the tool for the intended user groups.

3. Exploring whether the tool can take account of how methods are customised and adapted to project needs.

4. Extending the tool with additional functionalities like saving the current project.

\section{References}

1. BCS SIGiST: Usability Guidelines (2006), http://www.testingstandards.co.uk/usability_guidelines.htm

2. Ferre, X., Bevan, N., Escobar, T.A.: UCD Method Selection with Usability Planner. In: Proceedings of NordiCHI 2010 (2010)

3. GNU. General Public License version 3 (2007), http: / /www.gnu.org/licenses/gpl.html

4. ISO/PAS 18152: A specification for the process assessment of human-system issues (2003)

5. ISO/TR 16982: Usability methods supporting human-centred design (2002)

6. Seffah, A., Metzker, E.: The obstacles and myths of usability and software engineering. Communications of the ACM 47(12), 71-76 (2004)

7. Usability Planner tool (2011), http: / / usabilityplanner.org/ 